\title{
Algebraic Properties of First Integrals for Scalar Linear Third-Order ODEs of Maximal Symmetry
}

\author{
K. S. Mahomed and E. Momoniat \\ Centre for Differential Equations, Continuum Mechanics and Applications, School of Computational and Applied Mathematics, \\ University of the Witwatersrand, Wits 2050, South Africa
}

Correspondence should be addressed to K. S. Mahomed; komalmajeed@hotmail.com

Received 22 November 2012; Accepted 14 January 2013

Academic Editor: Emrullah Yaşar

Copyright ( 2013 K. S. Mahomed and E. Momoniat. This is an open access article distributed under the Creative Commons Attribution License, which permits unrestricted use, distribution, and reproduction in any medium, provided the original work is properly cited.

\begin{abstract}
By use of the Lie symmetry group methods we analyze the relationship between the first integrals of the simplest linear third-order ordinary differential equations (ODEs) and their point symmetries. It is well known that there are three classes of linear third-order ODEs for maximal cases of point symmetries which are 4, 5, and 7. The simplest scalar linear third-order equation has seven-point symmetries. We obtain the classifying relation between the symmetry and the first integral for the simplest equation. It is shown that the maximal Lie algebra of a first integral for the simplest equation $y^{\prime \prime \prime}=0$ is unique and four-dimensional. Moreover, we show that the Lie algebra of the simplest linear third-order equation is generated by the symmetries of the two basic integrals. We also obtain counting theorems of the symmetry properties of the first integrals for such linear third-order ODEs. Furthermore, we provide insights into the manner in which one can generate the full Lie algebra of higher-order ODEs of maximal symmetry from two of their basic integrals.
\end{abstract}

\section{Introduction}

Ordinary differential equations (ODEs) are a fertile area of study, especially the Lie algebraic properties of such equations and their first integrals have great importance. Initial investigations since the works of Lie [1] were motivated by physical problems, such as the free particle, one-dimensional harmonic oscillator, and Emden-Fowler equations and classification. Indeed, most of the earlier works dealt with secondorder linear equations (see, e.g., [2-8]). In the investigation by Mahomed and Leach [9], they found that the Lie point symmetries of the maximal cases of scalar linear $n$ th-order ODEs, $n \geq 3$, are $n+1, n+2$, and $n+4$. Thus for scalar linear third-order equations these correspond to 4,5 , and 7 symmetries. Moreover for scalar third-order linear ODEs, Govinder and Leach [10] provided the algebraic structure of the basic first integrals for such equations. They showed that the three equivalence classes each has certain first integrals with a specific number of point symmetries. They followed on the initial investigation of Leach and Mahomed [11] who considered the point symmetries of the basic first integrals of linear second-order ODEs. Then in the work [12] Flessas et al. attempted the symmetry structure for the first integrals of higher-order equations of maximal symmetry.

The subject of the present paper is the investigation of the Lie algebraic properties of first integrals of scalar linear third-order ODEs of the maximal class which is represented by $y^{\prime \prime \prime}=0$. We remind the reader that for the simplest class there has been some analysis made in Flessas et al. [12]. This is in regards to the maximal algebra possessed by an integral of $y^{\prime \prime \prime}=0$ which is listed in Table II of [12]. However, this is incomplete. We extend this study and provide a complete analysis on the Lie point symmetries and first integrals for the simplest third-order ODE including the maximal algebra case. We firstly deduce the classifying relation between the point symmetries and first integrals for this simple class. Then we use this to study the point symmetry properties of the first integrals of $y^{\prime \prime \prime}=0$ which also represents all linearizable by point transformations third-order ODEs that reduce to this class.

We begin by noting the condition for symmetries of the first integrals of scalar linear ODEs of order one. Then for 
completeness we review briefly the results of the paper by Mahomed and Momoniat [13] which discusses the relationship between the point symmetries of the first integrals of scalar linear second-order ODEs. These two cases are shown to be distinct in terms of their algebraic properties of their integrals when compared to higher-order ODEs of maximal symmetry.

1.1. Linear First-Order Equations. Consider the simplest firstorder ODE

$$
y^{\prime}=0
$$

It is easy to see that

$$
X=\xi(x, y) \frac{\partial}{\partial x}+\eta(x, y) \frac{\partial}{\partial y}
$$

is a point symmetry generator of (1) if

$$
\left.X^{[1]} y^{\prime}\right|_{y^{\prime}=0}=0
$$

where

$$
X^{[1]}=\xi \frac{\partial}{\partial x}+\eta \frac{\partial}{\partial y}+\zeta_{x} \frac{\partial}{\partial y^{\prime}}
$$

with

$$
\zeta_{x}=D_{x}(\eta)-y^{\prime} D_{x}(\xi)
$$

in which $D_{x}$ is the total differentiation operator and $X^{[1]}$ is the first prolongation of the generator $X$. We quickly see that

$$
\eta_{x}=0, \quad \eta=\eta(y)
$$

where $\eta$ is an arbitrary function of $y$. Therefore,

$$
X=\xi(x, y) \frac{\partial}{\partial x}+\eta(y) \frac{\partial}{\partial y} .
$$

Thus there is an infinite number of point symmetries. We now show that only $X=\xi(x, y) \partial / \partial x$ are symmetries of the first integral.

This forms an infinite-dimensional subalgebra of the Lie algebra of (1).

Now $I=y$ is a first integral of (1). It has point symmetry $X$ as in (2) if

$$
X I=0 .
$$

This implies that $\eta=0$ which immediately results in

$$
X=\xi(x, y) \frac{\partial}{\partial x} .
$$

There is an infinite number of symmetries of the first integral $I=y$ of (1).

Let $F$ be an arbitrary function of $I$, namely, $F=F(I)$. The symmetry of this general function of the first integral is

$$
X F=X I \frac{\partial F}{\partial I}=0 .
$$

Therefore $X$ as in (9) is a symmetry of $I=y$ and also any function of $F(y)$. Since any scalar first-order ODE is equivalent to the simplest one (1), this means that a first integral of a nonlinear first-order ODE has infinitely many symmetries too.

As an example, we consider the nonlinear first-order Riccati equation

$$
y^{\prime}+y^{2}=0
$$

the first integral of which is

$$
I=\frac{1}{y}-x .
$$

A symmetry of the first integral (12) is

$$
X=\frac{\partial}{\partial x}-y^{2} \frac{\partial}{\partial y} .
$$

In fact, we have an infinite number of symmetries given by

$$
X=\xi(x, y)\left(\frac{\partial}{\partial x}-y^{2} \frac{\partial}{\partial y}\right),
$$

where $\xi$ is an arbitrary function in its arguments.

Therefore we note here that the symmetries of the first integrals of a first-order equation form a proper subalgebra of the Lie algebra of the equation itself. We cannot generate the full algebra as is the case for linear scalar second-order ODEs [11] by use of the algebra of the basic integral alone.

The symmetries of the first integrals of scalar linear second-order ODEs have interesting properties [11, 13]. The first integrals of such linear equations can have $0,1,2$, and the maximum 3 symmetries [13]. The Lie algebra of the maximum case is unique. Peculiar to such equations is the other remarkable property that their Lie algebra is generated by the symmetry properties of the basic integrals and their quotient [11].

Below we study the symmetry properties of first integral for the simplest scalar linear third-order ODEs of maximal point symmetry. In the case of the basic first integrals, the algebraic properties are known from the work [10]. Here we pursue the relationship between symmetries and first integrals of scalar linear third-order ODEs for the simplest and maximal class. We obtain the classifying relation for this class and invoke this to arrive at counting theorems and the result on the maximal case of symmetries of the first integrals.

In the following we look at algebraic properties of first integrals for the seven point symmetry case by deriving the classifying relation between the symmetries and their first integrals. We use this relation to arrive at interesting properties which appear for the first time in the literature.

\section{Algebraic Properties of the First Integrals of $y^{\prime \prime \prime}=0$}

We consider the simplest third-order ODE

$$
y^{\prime \prime \prime}=0
$$


which as is well known has the seven symmetries (Lie [1] and e.g., [4])

$$
\begin{gathered}
X_{1}=\frac{\partial}{\partial y}, \quad X_{2}=x \frac{\partial}{\partial y}, \\
X_{3}=x^{2} \frac{\partial}{\partial y}, \quad X_{4}=y \frac{\partial}{\partial y}, \quad X_{5}=\frac{\partial}{\partial x}, \\
X_{6}=x \frac{\partial}{\partial x}+y \frac{\partial}{\partial y}, \quad X_{7}=x^{2} \frac{\partial}{\partial x}+2 x y \frac{\partial}{\partial y} .
\end{gathered}
$$

We have listed the symmetries in the order of the solution symmetries being first, then the homogeneity symmetry, and the remaining three which form the algebra $s l(2, R)$. This ODE (15) also represents all linearizable third-order ODEs reducible to it via point transformation. The order in which the symmetries appear in (16) is used in what follows. It is obvious that (15) has three functionally independent first integrals

$$
\begin{gathered}
I_{1}=y^{\prime \prime}, \\
I_{2}=x y^{\prime \prime}-y^{\prime}, \\
I_{3}=\frac{1}{2} x^{2} y^{\prime \prime}-x y^{\prime}+y .
\end{gathered}
$$

We use the ordering of the integrals as given in [10]. The first integral (17a) has four symmetries [10]

$$
\begin{gathered}
X_{1}=\frac{\partial}{\partial x}, \\
X_{2}=\frac{\partial}{\partial y}, \\
X_{3}=x \frac{\partial}{\partial y}, \\
X_{4}=x \frac{\partial}{\partial x}+2 y \frac{\partial}{\partial y},
\end{gathered}
$$

from which we observe that there are two solution symmetries, one translation in $x$ symmetry and a scaling symmetry. The translation in $x$ symmetry is a subset of the $\operatorname{sl}(2, R)$ symmetries with $X_{4}$ being a combination of the uniform scaling symmetry in both variables contained in the $s l(2, R)$ symmetries together with the homogeneity symmetry. Part of this fact was also noted in [12]. The second first integral (17b) has three symmetries [10]

$$
\begin{gathered}
Y_{1}=\frac{\partial}{\partial y}, \\
Y_{2}=x^{2} \frac{\partial}{\partial y}, \\
Y_{3}=x \frac{\partial}{\partial x}+y \frac{\partial}{\partial y},
\end{gathered}
$$

with two solution symmetries and $Y_{3}$ being part of the $s l(2, R)$ symmetries. The third first integral (17c) also has four symmetries [10]

$$
\begin{aligned}
& G_{1}=x \frac{\partial}{\partial x}, \\
& G_{2}=x \frac{\partial}{\partial y}, \\
& G_{3}=x^{2} \frac{\partial}{\partial y}, \\
& G_{4}=x^{2} \frac{\partial}{\partial x}+2 x y \frac{\partial}{\partial y} .
\end{aligned}
$$

Again one can see the solution symmetries, scaling, and the symmetry $G_{4}$ which is contained in the $s l(2, R)$ symmetries. Note further that the symmetries in (20) are found by multiplying those of (18) by the factor $x$. In fact these two sets are equivalent via a point transformation [10]. The other important properties are discussed in the next section.

Below we obtain the classifying relation.

2.1. Classifying Relation for the Symmetries and Integrals. Now let $F$ be an arbitrary function of the integrals (17a), (17b), and (17c), $I_{1}, I_{2}$, and $I_{3}$, namely,

$$
F=F\left(I_{1}, I_{2}, I_{3}\right) .
$$

The symmetry of this general function of the first integrals is

$$
X^{[2]} F=X^{[2]} I_{1} \frac{\partial F}{\partial I_{1}}+X^{[2]} I_{2} \frac{\partial F}{\partial I_{2}}+X^{[2]} I_{3} \frac{\partial F}{\partial I_{3}}=0,
$$

where

$$
\begin{aligned}
X^{[2]} I_{1}= & {\left[\xi \frac{\partial}{\partial x}+\eta \frac{\partial}{\partial y}+\zeta_{x} \frac{\partial}{\partial y^{\prime}}+\zeta_{x x} \frac{\partial}{\partial y^{\prime \prime}}\right] y^{\prime \prime} } \\
= & \zeta_{x x}, \\
X^{[2]} I_{2}= & {\left[\xi \frac{\partial}{\partial x}+\eta \frac{\partial}{\partial y}+\zeta_{x} \frac{\partial}{\partial y^{\prime}}+\zeta_{x x} \frac{\partial}{\partial y^{\prime \prime}}\right]\left(x y^{\prime \prime}-y^{\prime}\right) } \\
= & \xi y^{\prime \prime}-\zeta_{x}+x \zeta_{x x}, \\
X^{[2]} I_{3}= & {\left[\xi \frac{\partial}{\partial x}+\eta \frac{\partial}{\partial y}+\zeta_{x} \frac{\partial}{\partial y^{\prime}}+\zeta_{x x} \frac{\partial}{\partial y^{\prime \prime}}\right] } \\
& \times\left(\frac{1}{2} x^{2} y^{\prime \prime}-x y^{\prime}+y\right) \\
= & \xi\left(x y^{\prime \prime}-y^{\prime}\right)+\eta-x \zeta_{x}+\frac{1}{2} x^{2} \zeta_{x x} .
\end{aligned}
$$

The coefficient functions $\xi, \eta, \zeta_{x}$, and $\zeta_{x x}$ are

$$
\begin{gathered}
\xi=a_{5}+x a_{6}+x^{2} a_{7}, \\
\eta=a_{1}+x a_{2}+x^{2} a_{3}+y a_{4}+y a_{6}+2 x y a_{7}, \\
\zeta_{x}=a_{2}+2 x a_{3}+y^{\prime} a_{4}+2 y a_{7}, \\
\zeta_{x x}=2 a_{3}+y^{\prime \prime} a_{4}-y^{\prime \prime} a_{6}+\left(2 y^{\prime}-2 x y^{\prime \prime}\right) a_{7} .
\end{gathered}
$$


These are obtained by setting

$$
X^{[2]}=\sum_{i=1}^{7} a_{i} X_{i}^{[2]},
$$

where the $X_{i}$ are the symmetry generators as given in (16) and the $a_{i}$ are constants. The reason being that the symmetries of the first integrals are always the symmetries of the equation (see [14]).

After substitution of the values of $X^{[2]} I_{1}, X^{[2]} I_{2}$, and $X^{[2]} I_{3}$ as in (23), with $\xi, \eta, \zeta_{x}$, and $\zeta_{x x}$ given in (24), as well as by use of the first integrals $I_{1}=y^{\prime \prime}, I_{2}=x y^{\prime \prime}-y^{\prime}$, and $I_{3}=(1 / 2) x^{2} y^{\prime \prime}-x y^{\prime}+y$ in (22), we arrive at the classifying relation

$$
\begin{aligned}
{\left[2 a_{3}\right.} & \left.+\left(a_{4}-a_{6}\right) I_{1}-2 a_{7} I_{2}\right] \frac{\partial F}{\partial I_{1}} \\
& +\left(-a_{2}+a_{4} I_{2}+a_{5} I_{1}-2 a_{7} I_{3}\right) \frac{\partial F}{\partial I_{2}} \\
& +\left[a_{1}+\left(a_{4}+a_{6}\right) I_{3}+a_{5} I_{2}\right] \frac{\partial F}{\partial I_{3}}=0 .
\end{aligned}
$$

The relation (26) explicitly provides the relationship between the symmetries and the first integrals of the simple thirdorder equation (15). We invoke this to classify the first integrals according to their symmetries in what follows.

We use the classifying relation (26) to establish the number and property of symmetries possessed by the first integrals of the simplest third-order equation (15).

There arise five cases. We deal with each below.

Case 1 (no symmetry). If $F$ is any arbitrary function of $I_{1}, I_{2}$, and $I_{3}$, then $F_{I_{1}}, F_{I_{2}}$, and $F_{I_{3}}$ are not related to each other. In this case we have from (26) that

$$
\begin{gathered}
2 a_{3}+\left(a_{4}-a_{6}\right) I_{1}-2 a_{7} I_{2}=0, \\
-a_{2}+a_{4} I_{2}+a_{5} I_{1}-2 a_{7} I_{3}=0, \\
a_{1}+\left(a_{4}+a_{6}\right) I_{3}+a_{5} I_{2}=0 .
\end{gathered}
$$

It is easy to see from (27) that all the $a$ 's are zero. Therefore there exists no symmetry for this case.

As an illustrative example, if we take $F=I_{1} I_{2} \ln I_{3}$, then (26) straightforwardly yields

$$
\begin{aligned}
{\left[2 a_{3}+\right.} & \left.\left(a_{4}-a_{6}\right) I_{1}-2 a_{7} I_{2}\right] I_{2} I_{3} \ln I_{3} \\
& +\left(-a_{2}+a_{4} I_{2}+a_{5} I_{1}-2 a_{7} I_{3}\right) I_{1} I_{3} \ln I_{3} \\
& +\left[a_{1}+\left(a_{4}+a_{6}\right) I_{3}+a_{5} I_{2}\right] I_{1} I_{2}=0 .
\end{aligned}
$$

This easily results in all the $a$ 's being zero.

Case 2 (one symmetry). If $F$ satisfies the relation (26), then there exists one symmetry. For the simple symmetries of (15) one obtains further symmetries except for $X_{6}$ which we consider below.
If we take $F=I_{1} I_{2} I_{3}$ or any function of this product, then the relation (26) becomes

$$
\begin{aligned}
{\left[2 a_{3}\right.} & \left.+\left(a_{4}-a_{6}\right) I_{1}-2 a_{7} I_{2}\right] I_{2} I_{3} \\
& +\left(-a_{2}+a_{4} I_{2}+a_{5} I_{1}-2 a_{7} I_{3}\right) I_{1} I_{3} \\
& +\left[a_{1}+\left(a_{4}+a_{6}\right) I_{3}+a_{5} I_{2}\right] I_{1} I_{2}=0 .
\end{aligned}
$$

In (29), $a_{1}$ to $a_{7}$ are zeros except $a_{6}$ which gives the one symmetry

$$
X_{6}=x \frac{\partial}{\partial x}+y \frac{\partial}{\partial y} \text {. }
$$

Consider $J=I_{1} I_{3}-(1 / 2) I_{2}^{2}$ and $I_{2}=x y^{\prime \prime}-y^{\prime}$. Nowlet $F=J / I_{2}$ (cf. [12]). Then relation (26) becomes

$$
\begin{aligned}
{\left[2 a_{3}\right.} & \left.+\left(a_{4}-a_{6}\right) I_{1}-2 a_{7} I_{2}\right] 2 I_{2} I_{3} \\
& +\left(-a_{2}+a_{4} I_{2}+a_{5} I_{1}-2 a_{7} I_{3}\right)\left(-2 I_{1} I_{3}-I_{2}^{2}\right) \\
& +\left[a_{1}+\left(a_{4}+a_{6}\right) I_{3}+a_{5} I_{2}\right] 2 I_{1} I_{2}=0 .
\end{aligned}
$$

We see here that all the $a$ 's are zero except $a_{6}$. Therefore there exists one symmetry which again is (30).

In fact similar to the free particle equation [13], there are many one symmetry cases.

Case 3 (two symmetries). Here there are many cases as well. We begin by utilizing the Lie table for the classification of the two-dimensional algebras in the plane which are given, for example, in [6]. They are

$$
\begin{gathered}
Y_{1}=\frac{\partial}{\partial y}, \quad Y_{2}=\frac{\partial}{\partial x}, \\
Y_{1}=\frac{\partial}{\partial y}, \quad Y_{2}=x \frac{\partial}{\partial y}, \\
Y_{1}=\frac{\partial}{\partial y}, \quad Y_{2}=x \frac{\partial}{\partial x}+y \frac{\partial}{\partial y}, \\
Y_{1}=\frac{\partial}{\partial y}, \quad Y_{2}=y \frac{\partial}{\partial y} .
\end{gathered}
$$

These form subalgebras of the Lie algebra of symmetries of (15) as can clearly be observed.

We take the first realization listed above. If $a_{1}$ is arbitrary in (26), then $X_{1}=\partial / \partial y$ implies that $F$ is independent of $I_{3}$. Further $X_{5}=\partial / \partial x$ yields that $F$ does not depend on $I_{2}$ as well. Since we require that $\partial F / \partial I_{1} \neq 0$, we have

$$
2 a_{3}+I_{1}\left(a_{4}-a_{6}\right)-2 a_{7} I_{2}=0,
$$

from which it follows that $a_{3}=a_{7}=0$ and $a_{4}=a_{6}$. Thus we end up with two more symmetries $X_{2}$ and $X_{4}+X_{6}$. These turn out to be the four symmetries of the integral $I_{1}$ given in (18).

Likewise for the second realization we obtain (18) again. For the third realization listed above we find the symmetries of $I_{2}$ as in (19). 
Hence the first three realizations listed do not provide maximal two symmetries for the first integrals of (15).

In fact the fourth realization results in a two symmetry case as $a_{1}$ and $a_{4}$ are arbitrary and give rise to

$$
\frac{\partial F}{\partial I_{3}}=0, \quad I_{1} \frac{\partial F}{\partial I_{1}}+I_{2} \frac{\partial F}{\partial I_{2}}=0,
$$

which has the solution

$$
F=H \frac{I_{2}}{I_{1}}
$$

The further substitution of this form into the relation (26) constrains all the $a$ 's to be zero except for $a_{1}$ and $a_{4}$. This result prompts the following simple products and quotients that do give two symmetries.

If $F=I_{1} I_{2}$, then relation (26) yields

$$
\begin{aligned}
{\left[2 a_{3}\right.} & \left.+\left(a_{4}-a_{6}\right) I_{1}-2 a_{7} I_{2}\right] I_{2} \\
& +\left(-a_{2}+a_{4} I_{2}+a_{5} I_{1}-2 a_{7} I_{3}\right) I_{1}=0
\end{aligned}
$$

Here we observe that $a_{2}, a_{3}, a_{5}$, and $a_{7}$ are zeros whereas $a_{1}$ is arbitrary and $a_{6}=2 a_{4}$, and therefore we obtain the two symmetries

$$
\begin{gathered}
X_{1}=\frac{\partial}{\partial y} \\
Y=2 x \frac{\partial}{\partial x}+3 y \frac{\partial}{\partial y}
\end{gathered}
$$

which form a two-dimensional algebra with

$$
\left[X_{1}, Y\right]=3 X_{1} \text {. }
$$

If we set $F=I_{1} I_{3}$, then we end up getting $a_{1}, a_{3}, a_{4}, a_{5}$, and $a_{7}$ equal to zero. Since $a_{2}$ and $a_{6}$ are arbitrary so they result in two symmetries

$$
\begin{gathered}
X_{2}=x \frac{\partial}{\partial y} \\
X_{6}=x \frac{\partial}{\partial x}+y \frac{\partial}{\partial y}
\end{gathered}
$$

with Lie bracket

$$
\left[X_{2}, X_{6}\right]=0
$$

If we take $F=I_{2} I_{3}$, then we see that $a_{3}$ is arbitrary and $a_{6}=$ $-2 a_{4}$ which then give rise to the two symmetries

$$
\begin{gathered}
X_{3}=x^{2} \frac{\partial}{\partial y} \\
Y=2 x \frac{\partial}{\partial x}+y \frac{\partial}{\partial y},
\end{gathered}
$$

with

$$
\left[X_{3}, Y\right]=-3 X_{3}
$$

Consider now $F=I_{3} / I_{1}$. This shows that $a_{2}$ and $a_{4}$ are arbitrary, and the resulting two symmetries are

$$
\begin{aligned}
& X_{2}=x \frac{\partial}{\partial y}, \\
& X_{4}=y \frac{\partial}{\partial y},
\end{aligned}
$$

with commutation relation

$$
\left[X_{2}, X_{4}\right]=X_{2}
$$

If we let $F=I_{3} / I_{2}$, then here $a_{3}$ and $a_{4}$ are arbitrary, and therefore the two symmetries are

$$
\begin{aligned}
& X_{3}=x^{2} \frac{\partial}{\partial y} \\
& X_{4}=y \frac{\partial}{\partial y}
\end{aligned}
$$

with

$$
\left[X_{3}, X_{4}\right]=X_{3}
$$

If $J=I_{1} I_{3}-(1 / 2) I_{2}^{2}, I_{1}=y^{\prime \prime}$, and $F=J / I_{1}$ (cf. [12]), then we have from the relation (26)

$$
\begin{aligned}
{\left[2 a_{3}\right.} & \left.+\left(a_{4}-a_{6}\right) I_{1}-2 a_{7} I_{2}\right] \frac{1}{2} I_{2}^{2} \\
& +\left[-a_{2}+a_{4} I_{2}+a_{5} I_{1}-2 a_{7} I_{3}\right]\left(-I_{1} I_{2}\right) \\
& +\left[a_{1}+\left(a_{4}+a_{6}\right) I_{3}+a_{5} I_{2}\right] I_{1}^{2}=0 .
\end{aligned}
$$

This results in $a_{1}, a_{2}, a_{3}$, and $a_{7}$ being zero whereas $a_{5}$ is arbitrary, and $a_{4}=-a_{6}$, which give rise to the two symmetries

$$
\begin{aligned}
& X_{5}=\frac{\partial}{\partial x} \\
& Y=x \frac{\partial}{\partial x}
\end{aligned}
$$

with

$$
\left[X_{5}, Y\right]=X_{5}
$$

If $J=I_{1} I_{3}-(1 / 2) I_{2}^{2}, I_{3}=(1 / 2) x^{2} y^{\prime \prime}-x y^{\prime}+y$, and $F=J / I_{3}$ (cf. [12]), then relation (26) yields

$$
\begin{aligned}
{\left[2 a_{3}\right.} & \left.+\left(a_{4}-a_{6}\right) I_{1}-2 a_{7} I_{2}\right] 2 I_{3}^{2} \\
& +\left(-a_{2}+a_{4} I_{2}+a_{5} I_{1}-2 a_{7} I_{3}\right)\left(-2 I_{3} I_{2}\right) \\
& +\left[a_{1}+\left(a_{4}+a_{6}\right) I_{3}+a_{5} I_{2}\right] I_{2}^{2}=0 .
\end{aligned}
$$

The above relation shows that $a_{1}, a_{2}, a_{3}$, and $a_{5}$ are zeros, $a_{7}$ is arbitrary, and $a_{4}=a_{6}$. This imply two symmetries

$$
\begin{gathered}
X_{7}=x^{2} \frac{\partial}{\partial x}+2 x y \frac{\partial}{\partial y}, \\
Y=x \frac{\partial}{\partial x}+2 y \frac{\partial}{\partial y}
\end{gathered}
$$


together with

$$
\left[X_{7}, Y\right]=-X_{7}
$$

There are thus many two symmetry cases. One could obtain more. Also they could arise as different combinations of the seven symmetries (16). So one concludes that the twodimensional algebra cases are not unique. We have seen the occurrence of both Abelian and non-Abelian Lie algebras.

Case 4 (three symmetries). Here we use the three-dimensional real realizations of Mahomed and Leach [7]. The notation used is that given in [4]. Since we adapt these realizations as symmetries of third-order equations, the entries for the first entry $L_{3 ; 1}$ and the nonsolvable algebra $L_{3 ; 8}^{\mathrm{I}}$ are those which are symmetries of such equations.

Note that for $L_{3 ; 8}^{\mathrm{II}}, L_{3 ; 8}^{\mathrm{III}}$, and $L_{3 ; 8}^{\mathrm{IV}}$ one can use the realizations as given in Table 1 or the ones obtained by interchanging $x$ and $y$ in the realizations given. The reason for this is that one still obtains third-order representative equations for the latter realizations.

As $L_{3 ; 1}$ is the first three-dimensional algebra in Table 1 , we start with that. We want this Abelian algebra to be admitted by a first integral of (15). We utilize the classifying relation (26). Therefore $a_{1}, a_{2}$, and $a_{3}$ are arbitrary which imply that $F$ is constant. Hence this algebra is not admitted by any first integral of (15) although, if it is admitted by a nonlinear thirdorder ODE, it implies linearization (see Mahomed and Leach [6]).

It is not difficult to deduce that the same applies to the algebras $L_{3 ; 2}, L_{3 ; 3}^{\mathrm{I}}, L_{3 ; 3}^{\mathrm{II}}, L_{3 ; 4}^{\mathrm{I}}, L_{3 ; 4}^{\mathrm{II}}, L_{3 ; 5}^{\mathrm{I}}, L_{3 ; 5}^{\mathrm{II}}$, and $L_{3 ; 6}^{\mathrm{I}}$ when $a \neq 2$ and $L_{3 ; 6}^{\mathrm{II}}$ when $a \neq 1 / 2,-1$. If $a=2$ for $L_{3 ; 6}^{\mathrm{I}}$, then one ends up with four symmetries of $F=F\left(I_{1}\right)$ which are (18). The same applies to $L_{3 ; 6}^{\mathrm{II}}, a=1 / 2$. For both these cases, the algebras $L_{3 ; 6}^{\mathrm{I}}, a=2$ and $L_{3 ; 6}^{\mathrm{II}}, a=1 / 2$ are admitted by a first integral $F=F\left(I_{1}\right)$ but these are not maximal and contained in a four-dimensional algebra, spanned by operators (18).

Now we focus on the three symmetry case which is admitted by $F=F\left(I_{2}\right)$. These three symmetries are given in (19). The Lie algebra of the generators (19) has nonzero commutators

$$
\left[X_{1}, X_{3}\right]=X_{1}, \quad\left[X_{2}, X_{3}\right]=-X_{2},
$$

with the elements thus forming the Lie algebra $L_{3 ; 6}^{\mathrm{II}}, a=-1$. In fact the transformation

$$
X=x^{2}, \quad Y=x+y,
$$

maps it to the canonical form of Table 1, namely,

$$
\bar{X}_{1}=\frac{\partial}{\partial Y}, \quad \bar{X}_{2}=X \frac{\partial}{\partial Y}, \quad \bar{X}_{3}=2 X \frac{\partial}{\partial X}+Y \frac{\partial}{\partial Y} .
$$

Therefore the symmetries of $F=F\left(I_{2}\right)$ has the Lie algebra $L_{3 ; 6}^{\mathrm{II}}, a=-1$.

There is yet another three-dimensional algebra which is admitted by a first integral of (15). This occurs for $L_{3 ; 8}^{\mathrm{I}}$. We use
TABLE 1: Realizations of three-dimensional algebras in the real plane.

\begin{tabular}{ll}
\hline & $p=\partial / \partial x$ and $q=\partial / \partial y$ \\
\hline Algebra & Realizations in $(x, y)$ plane \\
\hline$L_{3 ; 1}$ & $X_{1}=q, X_{2}=x q, X_{3}=x^{2} q$ \\
$L_{3 ; 2}$ & $X_{1}=q, X_{2}=p, X_{3}=x q$ \\
$L_{3 ; 3}^{\mathrm{I}}$ & $X_{1}=q, X_{2}=p, X_{3}=x p+(x+y) q$ \\
$L_{3 ; 3}^{\mathrm{II}}$ & $X_{1}=q, X_{2}=x q, X_{3}=p+y q$ \\
$L_{3 ; 4}^{\mathrm{I}}$ & $X_{1}=p, X_{2}=q, X_{3}=x p$ \\
$L_{3 ; 4}^{\mathrm{II}}$ & $X_{1}=q, X_{2}=x q, X_{3}=x p+y q$ \\
$L_{3 ; 5}^{\mathrm{I}}$ & $X_{1}=p, X_{2}=q, X_{3}=x p+y q$ \\
$L_{3 ; 5}^{\mathrm{II}}$ & $X_{1}=q, X_{2}=x q, X_{3}=y q$ \\
$L_{3 ; 6}^{\mathrm{I}}$ & $X_{1}=p, X_{2}=q, X_{3}=x p+a y q, a \neq 0,1$ \\
$L_{3 ; 6}^{\mathrm{II}}$ & $X_{1}=q, X_{2}=x q, X_{3}=(1-a) x p+y q, a \neq 0,1$ \\
$L_{3 ; 7}^{\mathrm{I}}$ & $X_{1}=p, X_{2}=q, X_{3}=(b x+y) p+(b y-x) q$ \\
$L_{3 ; 7}^{\mathrm{II}}$ & $X_{1}=x q, X_{2}=q, X_{3}=\left(1+x^{2}\right) p+(x+b) y q$ \\
$L_{3 ; 8}^{\mathrm{I}}$ & $X_{1}=p, X_{2}=x p+y q, X_{3}=x^{2} p+2 x y q$ \\
$L_{3 ; 8}^{\mathrm{II}}$ & $X_{1}=q, X_{2}=x p+y q, X_{3}=2 x y p+\left(y^{2}-x^{2}\right) q$ \\
$L_{3 ; 8}^{\mathrm{III}}$ & $X_{1}=q, X_{2}=x p+y q, X_{3}=2 x y p+\left(y^{2}+x^{2}\right) q$ \\
$L_{3 ; 8}^{\mathrm{IV}}$ & $X_{1}=q, X_{2}=y q, X_{3}=y^{2} q$ \\
$L_{3 ; 9}$ & $X_{1}=\left(1+x^{2}\right) p+x y q, X_{2}=x y p+\left(1+y^{2}\right) q$, \\
& $X_{3}=y p-x q$ \\
\hline &
\end{tabular}

the classifying relation (26). Here $a_{5}, a_{6}$, and $a_{7}$ are arbitrary. Making these constants one at a time unity and the rest zero yield is

$$
\begin{aligned}
& I_{1} \frac{\partial F}{\partial I_{2}}+I_{2} \frac{\partial F}{\partial I_{3}}=0 \\
& -I_{1} \frac{\partial F}{\partial I_{1}}+I_{3} \frac{\partial F}{\partial I_{3}}=0 \\
& I_{2} \frac{\partial F}{\partial I_{1}}+I_{3} \frac{\partial F}{\partial I_{2}}=0 .
\end{aligned}
$$

The solution to this system (56) yields

$$
F=F\left(I_{1} I_{3}-\frac{1}{2} I_{2}^{2}\right) \text {. }
$$

Thus the basic first integral $J=I_{1} I_{3}-(1 / 2) I_{2}^{2}$ has the algebra $L_{3 ; 8}^{\mathrm{I}}$.

The Lie algebras $L_{3 ; 8}^{\mathrm{II}}, L_{3 ; 8}^{\mathrm{III}}$, and $L_{3 ; 8}^{\mathrm{IV}}$ are not admitted by any first integral of (15) as these are subalgebras of the maximal six-dimensional algebras (see Ibragimov and Mahomed [3]) which are admitted by nonlinear third-order ODEs not reducible to the simplest equation (15).

In the case of the algebra $L_{3 ; 9}$ one has the situation that this algebra is not a subalgebra of the seven-dimensional algebra of (15) (Wafo Soh and Mahomed [15]).

In conclusion of this discussion, we have two threedimensional algebras admitted by a first integral of (15) which are $L_{3 ; 8}^{\mathrm{I}}$ and $L_{3 ; 6}^{\mathrm{II}}, a=-1$. 
We state the following theorem.

Theorem 1. If a first integral of the simplest third-order ODE, $y^{\prime \prime \prime}=0$, admits a three-dimensional algebra, then it is one of the two three-dimensional algebras $L_{3 ; 6}^{I I}, a=-1$, or $L_{3 ; 8}^{I}$.

The proof follows from the previous discussions. Note here that the three-dimensional algebra admitted is not unique.

Case 5 (four symmetries). In the four symmetry case we have that only $I_{1}$ and $I_{3}$ possess four symmetries. They are given by (18) and (20). Both are similar by a point transformation [10]. The Lie algebra of (18) is $L_{4 ; 9}^{\mathrm{I}}$ (see [12]). This can be seen by interchanging $X_{1}$ and $X_{2}$ in (18). Thus the nonzero commutation relations are

$$
\begin{array}{ll}
{\left[X_{2}, X_{3}\right]=X_{1},} & {\left[X_{1}, X_{4}\right]=2 X_{1},} \\
{\left[X_{2}, X_{4}\right]=X_{2},} & {\left[X_{3}, X_{4}\right]=X_{3} .}
\end{array}
$$

We have the following theorem.

Theorem 2. The maximum dimension of the Lie algebra admitted by any first integral of the simplest third-order ODE, $y^{\prime \prime \prime}=0$, or any third-order ODE linearizable by point transformation to the simplest ODE is $L_{4 ; 9}^{I}$.

Proof. Any first integral of $y^{\prime \prime \prime}=0$ or third-order ODE reducible to the simplest ODE by point transformation cannot admit a maximal algebra of dimension greater than four since the basic integrals $I_{1}$ and $I_{3}$ have the unique four dimension algebra $L_{4 ; 9}^{\mathrm{I}}$. The other integrals have lower dimensional Lie algebras in the classification obtained above.

Finally, we have the following counting theorem.

Theorem 3. A first integral of the simplest third-order ODE, $y^{\prime \prime \prime}=0$, or any linearizable third-order ODE by point transformation to the simplest ODE can have $0,1,2,3$, or the maximum 4 symmetries. The four symmetry case is unique.

\section{Symmetry Properties of First Integrals of Higher-Order ODEs: Some Remarks}

In the case of symmetries of the simplest first-order ODE $y^{\prime}=0$ we have seen that the algebra of any first integral constitutes a proper subalgebra of the equation itself. One cannot generate the full algebra of $y^{\prime}=0$ via the algebras of any integral. This result also applies to any scalar first-order ODE due to equivalence of this with the simplest equation.

What occurs for scalar linear second-order ODEs is very different to the first-order ODE case. Here as has been shown in [11], the Lie algebra of $y^{\prime \prime}=0$ which represents any linear or linearizable second-order ODE can be generated by the threedimensional algebras of the triplets of the basic integrals and their quotient which are isomorphic to each other. Thus in this case one requires not only the basic integrals, say $I_{1}$ and $I_{2}$, but also a functionally dependent quotient integral $J=$ $I_{2} / I_{1}$.
Another important point to make is that the full Lie algebra of the simplest third-order equation (15) is generated by the four symmetries (18) as well as the three symmetries $G_{2}$, $G_{3}$, and $G_{4}$ of (20). Hence one requires only the symmetries of the basic integrals $I_{1}$ and $I_{3}$ to generate the full algebra of our equation (15). One should contrast this with what happens for $y^{\prime}=0$ and $y^{\prime \prime}=0$ discussed above. So one has the seven symmetries of our equation (15) being generated by four symmetries of $I_{1}$ together with three symmetries of $I_{3}$. The natural question then is as follows: what occurs for higherorder ODEs of maximal symmetry? Patterns emerge, some of which are discussed in the three propositions in the paper of Flessas et al. [12]. We discuss another important property, namely, that of generation of the full algebra via integrals now.

Consider the $n$ th-order ODE of maximal symmetry

$$
y^{(n)}=0, \quad n \geq 3 .
$$

This ODE (59) has $n+4$ symmetries as is well known. The $n$ first integrals of (59) are easily constructible and we focus on the first and last which are

$$
\begin{gathered}
I_{1}=y^{(n-1)}, \\
I_{n}=\sum_{i=1}^{n} \frac{(-1)^{i-1}}{(n-i) !} x^{n-i} y^{(n-i)} .
\end{gathered}
$$

The first integral (60) has $n+1$ symmetries which are

$$
\begin{gathered}
X_{i}=x^{i-1} \frac{\partial}{\partial y}, \quad i=1, \ldots, n-1, \\
X_{n}=\frac{\partial}{\partial x} \\
X_{n+1}=x \frac{\partial}{\partial x}+(n-1) y \frac{\partial}{\partial y} .
\end{gathered}
$$

This forms an $n+1$-dimensional subalgebra of (59). Now the first integral (61) has symmetries

$$
\begin{gathered}
Y_{i}=x^{i} \frac{\partial}{\partial y}, \quad i=1, \ldots, n-1, \\
Y_{n}=x \frac{\partial}{\partial x}, \\
Y_{n+1}=x^{2} \frac{\partial}{\partial x}+(n-1) x y \frac{\partial}{\partial y},
\end{gathered}
$$

which one can see comes from multiplying the symmetries of (62) by $x$. We can observe from these two sets (62) and (63) that the full Lie algebra of our equation (59) is generated from the $n+1$ symmetries of (62) and 3 symmetries of (63), namely, $Y_{n-1}, Y_{n}$, and $Y_{n+1}$. Further the two sets (62) and (63) are equivalent to each other by means of the point transformation

$$
\bar{x}=\frac{1}{x}, \quad \bar{y}=\frac{y}{x^{n-1}} .
$$

This is an extension of the transformation given in [10] and is for higher-order ODEs. In [10] it was given for third-order ODEs. 
We have the following theorem, the proof of which is evident from the above.

Theorem 4. The full Lie algebra of the nth-order ODE $y^{(n)}=$ $0, n \geq 3$, is generated by two subalgebras, namely, the $n+1$ dimensional algebra $\left\langle X_{j}: j=1, \ldots, n+1\right\rangle$ of $I_{1}$ and the threedimensional subalgebra $\left\langle Y_{n-1}, Y_{n}, Y_{n+1}\right\rangle$ of $I_{n}$.

Hence the picture is quite distinct for the manner in which the full Lie algebra is generated for the ODEs $y^{\prime}=0$, $y^{\prime \prime}=0$ and, $y^{(n)}=0, n \geq 3$. This is also consistent with the properties of their symmetry algebra which are different (see, e.g., [4]).

\section{Acknowledgments}

K. S. Mahomed is thankful to the University of the Witwatersrand and the NRF (South Africa) for financial support. E. Momoniat thanks the NRF (South Africa) for financial support.

\section{References}

[1] S. Lie, "Klassifikation und Integration von gewöhnlichen differentialgleichungen zwischen $x, y$, die eine Gruppe von Transformationen gestatten," Archiv der Mathematik, vol. 8, no. 9, p. 187, 1883.

[2] P. G. L. Leach, "First integrals for the modified Emden equation $\ddot{q}+\alpha(t) \dot{q}+q^{n}=0$," Journal of Mathematical Physics, vol. 26, no. 10 , pp. $2510-2514,1985$.

[3] N. H. Ibragimov and F. M. Mahomed, "Ordinary differential equations," in CRC Handbook of Lie Group Analysis of Differential Equations, N. H. Ibragimov, Ed., vol. 3, p. 191, CRC Press, Boca Raton, Fla, USA, 1996.

[4] F. M. Mahomed, "Symmetry group classification of ordinary differential equations: survey of some results," Mathematical Methods in the Applied Sciences, vol. 30, no. 16, pp. 1995-2012, 2007.

[5] F. M. Mahomed and P. G. L. Leach, "The linear symmetries of a nonlinear differential equation," Quaestiones Mathematicae, vol. 8, no. 3, pp. 241-274, 1985.

[6] F. M. Mahomed and P. G. L. Leach, "Lie algebras associated with scalar second-order ordinary differential equations," Journal of Mathematical Physics, vol. 30, no. 12, pp. 2770-2777, 1989.

[7] F. M. Mahomed and P. G. L. Leach, "The Lie algebra sl $(3, R)$ and linearization," Quaestiones Mathematicae, vol. 12, no. 2, pp. 121139, 1989.

[8] F. M. Mahomed, A. H. Kara, and P. G. L. Leach, "Lie and Noether counting theorems for one-dimensional systems," Journal of Mathematical Analysis and Applications, vol. 178, no. 1, pp. 116129, 1993.

[9] F. M. Mahomed and P. G. L. Leach, "Symmetry Lie algebras of $n$th order ordinary differential equations," Journal of Mathematical Analysis and Applications, vol. 151, no. 1, pp. 80-107, 1990.

[10] K. S. Govinder and P. G. L. Leach, "The algebraic structure of the first integrals of third-order linear equations," Journal of Mathematical Analysis and Applications, vol. 193, no. 1, pp. 114133, 1995.

[11] P. G. L. Leach and F. M. Mahomed, "Maximal subalgebra associated with a first integral of a system possessing $\operatorname{sl}(3, R)$ algebra," Journal of Mathematical Physics, vol. 29, no. 8, pp. 1807$1813,1988$.

[12] G. P. Flessas, K. S. Govinder, and P. G. L. Leach, "Characterisation of the algebraic properties of first integrals of scalar ordinary differential equations of maximal symmetry," Journal of Mathematical Analysis and Applications, vol. 212, no. 2, pp. 349-374, 1997.

[13] K. S. Mahomed and E. Momoniat, "Symmetry classiffcation of first integrals for scalar linearizable second-order ODEs," Journal of Applied Mathematics, vol. 2012, Article ID 847086, 14 pages, 2012.

[14] A. H. Kara and F. M. Mahomed, "A basis of conservation laws for partial differential equations," Journal of Nonlinear Mathematical Physics, vol. 9, supplement 2, pp. 60-72, 2002.

[15] C. Wafo Soh and F. M. Mahomed, "Contact symmetry algebras of scalar ordinary differential equations," Nonlinear Dynamics, vol. 28, no. 2, pp. 213-230, 2002. 


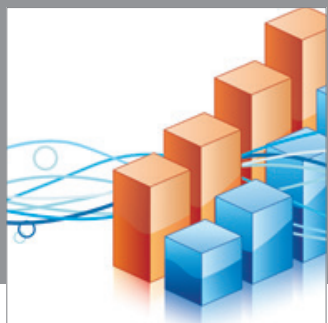

Advances in

Operations Research

mansans

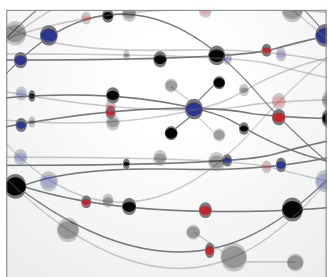

The Scientific World Journal
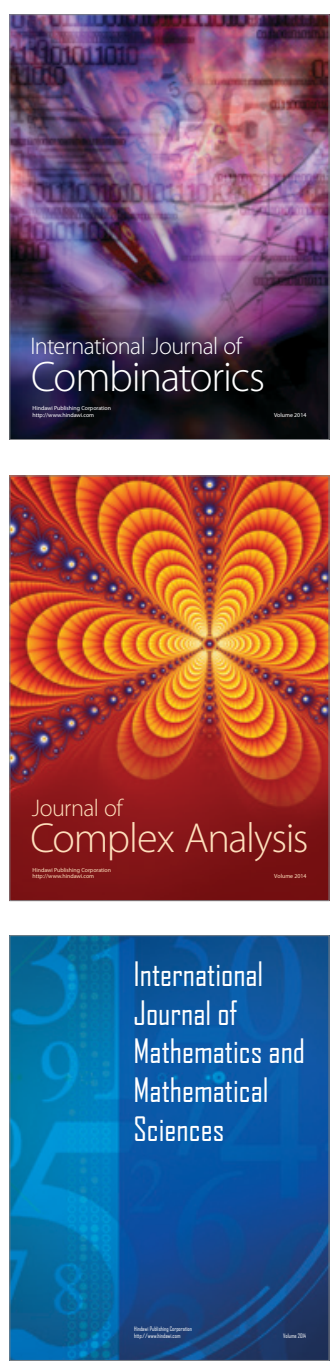
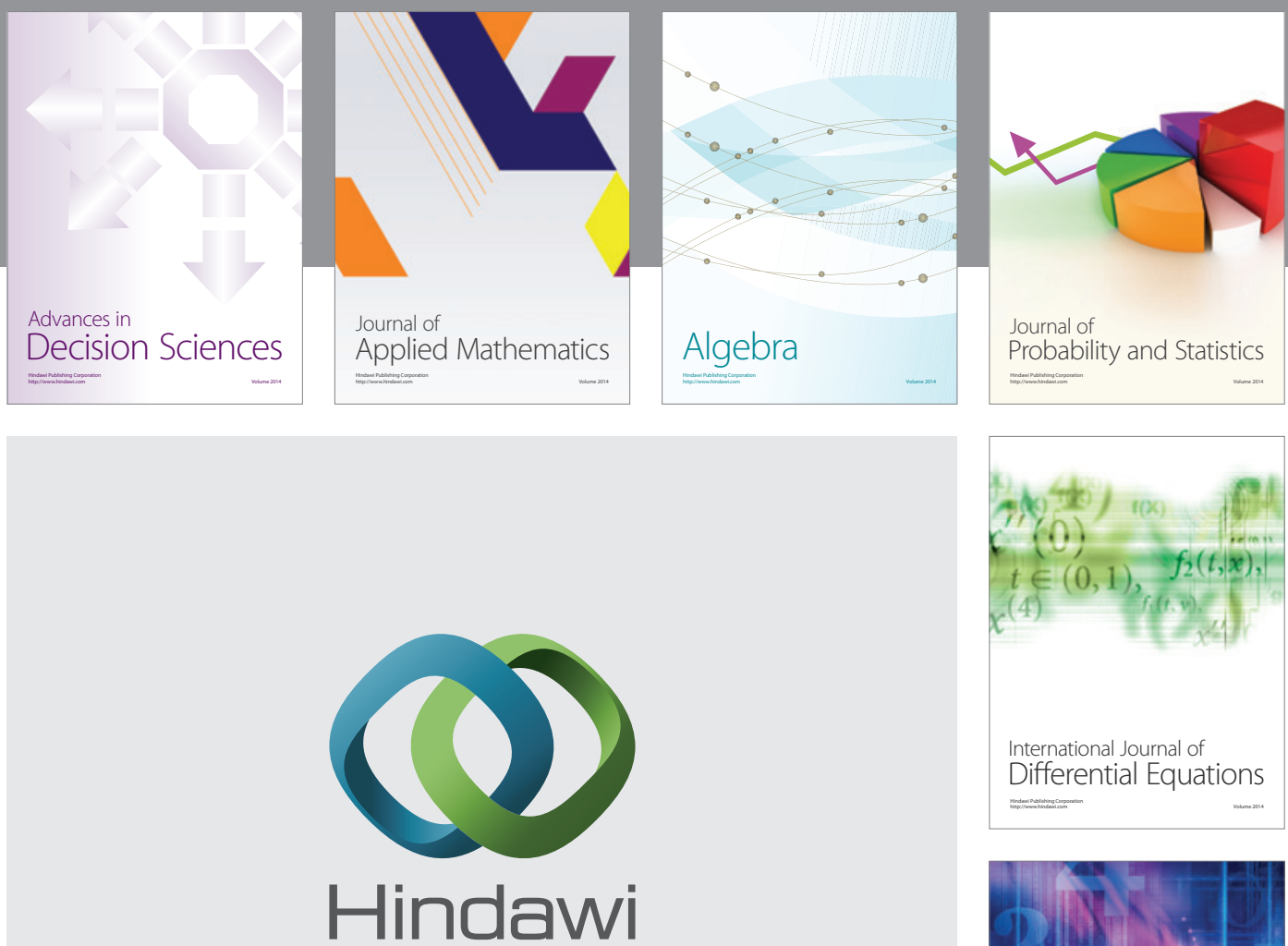

Submit your manuscripts at http://www.hindawi.com
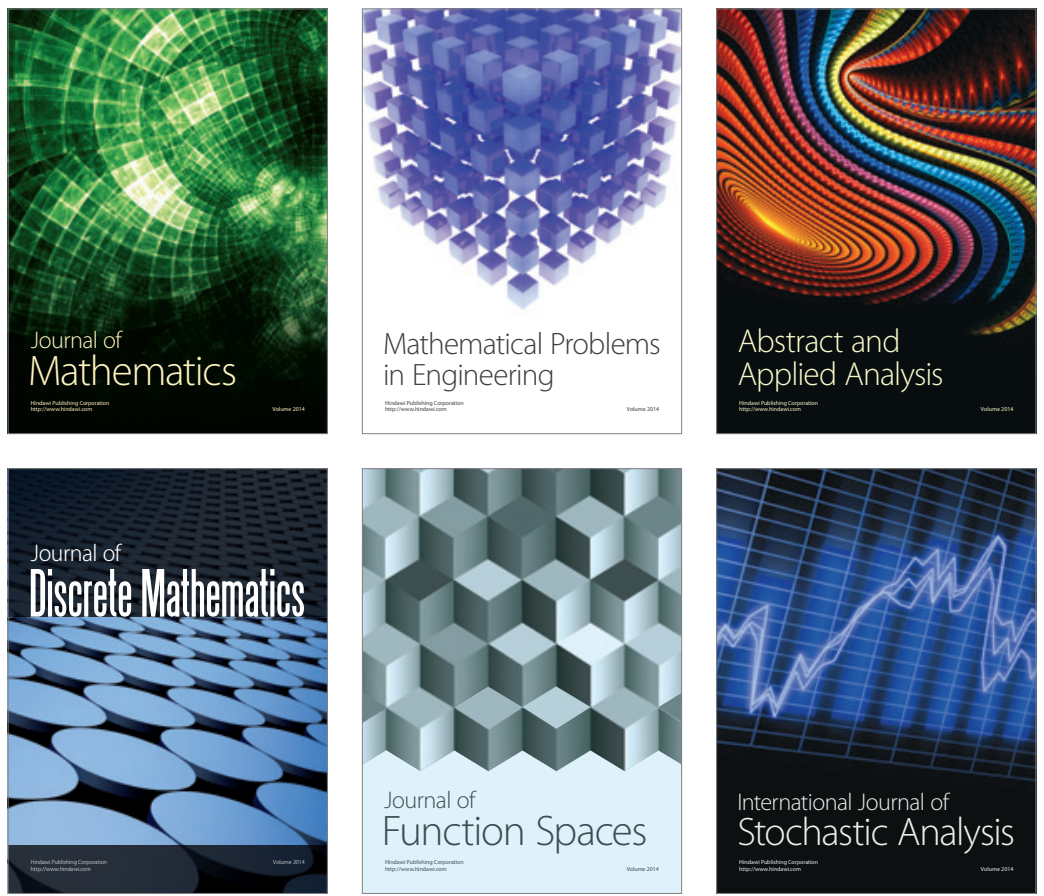

Journal of

Function Spaces

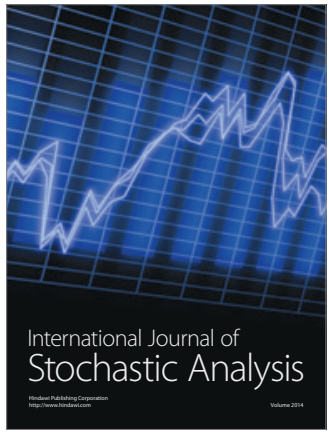

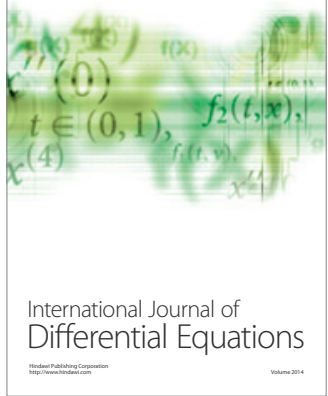
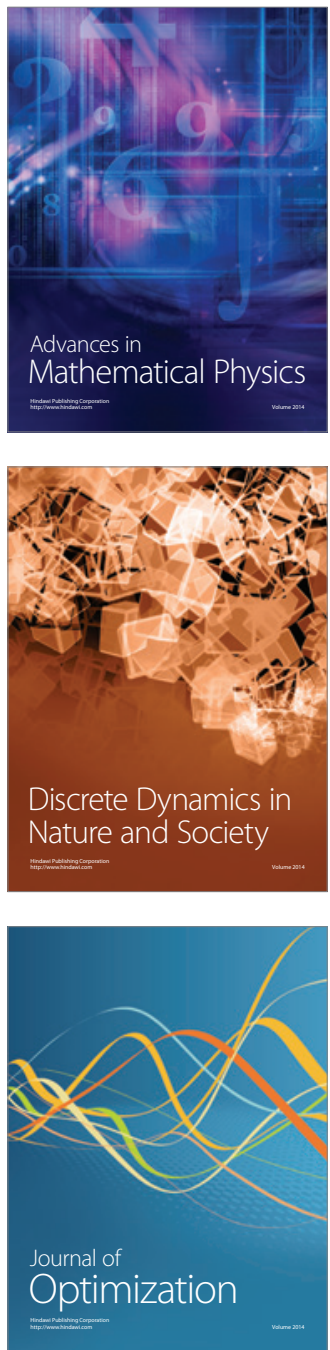